\title{
Determination of Johnson-holmquist constitutive model parameters for fused silica
}

\author{
A. Ruggiero ${ }^{1}$, G. lannitti ${ }^{1}$, N. Bonora ${ }^{1, a}$, and M. Ferraro ${ }^{2}$ \\ ${ }^{1}$ University of Cassino, DiMSAT, Cassino, Italy \\ ${ }^{2}$ MBDA Italia Spa, Bacoli, Italy
}

\begin{abstract}
Johnson-Holmquist constitutive model parameters for Fused Silica under dynamic conditions were determined by means of an inverse calibration technique. The identification of the parameters was performed with an multi-object optimizer using experimental data generated by two different validation tests: Taylor Cylinder Impact tests and Drop Weight tests, with impact velocity and strain rate ranging from 1 to $100 \mathrm{~m} / \mathrm{s}$ and from $10^{2}$ to $10^{4} \mathrm{~s}^{-1}$, respectively. The validity of the parameters set determined in this way was verified comparing numerical predictions and experimental results for an independent designed test, given by a fused silica tile impacted at prescribed velocity by a steel sphere.
\end{abstract}

\section{Introduction}

Fused silica is a high purity synthetic amorphous silicon dioxide characterized by low thermal expansion coefficient, excellent optical qualities and exceptional transmittance over a wide spectral range. Because of its wide use in the military industry as window material, it may be subjected to high-energy ballistic impacts. Under such dynamic conditions, post-yield response of the ceramic as well as the strain rate related effects become significant and should be accounted for in the constitutive modeling. Today, one of the most widely used constitutive model for simulating dynamic behavior of ceramic materials is the later version of the Johnson-Holmquist model [1], usually indicated as JH-2 ceramic model. In this study, for the identification of the model parameters, a procedure based on the inverse calibration technique of experimental validation tests was developed. To this purpose, Taylor impact tests and drop weight tests were designed and performed at different impact velocities ranging from 1 to $100 \mathrm{~m} / \mathrm{s}$ and a strain rate range ranging from $10^{2}$ up to $10^{4} \mathrm{~s}^{-1}$. Numerical simulation of both tests was performed with explicit finite element code. Identification of the model parameters was performed by means of structural optimization using as objective functions multiple validation metrics. The validity of the parameters set determined with the proposed procedure was verified comparing numerical predictions and experimental results for an independent designed test consisting in a fused silica tile impacted at prescribed velocity by a steel sphere.

\section{JH-2 Model parameters}

Ceramic materials are commonly used in protective armor applications and may be subject to high-energy ballistic impacts in these situations. Under simple loading conditions, ceramics may be regarded as elastic-brittle materials. However, when considering ballistic impacts, the postyield response of the ceramic becomes significant. One of

a e-mail: nbonora@unicas.it the most widely used constitutive models for simulating the post-yield response of ceramic materials is the JH-2 ceramic model. This constitutive equation, developed by Johnson and Holmquist, incorporates the effect of damage on residual material strength and bulking during failure in compression. The relevant equations describing the response of the material are summarized in the following. The strength of the material is described by a smoothly varying function of the intact strength, fractured strength, and damage:

$$
\sigma^{*}=\sigma_{i}^{*}-D\left(\sigma_{i}^{*}-\sigma_{f}^{*}\right)
$$

where the stresses are normalized respect to $\sigma_{H E L}$ that is defined as follows,

$$
\sigma_{H E L}=\frac{3}{2}\left(H E L-P_{H E L}\right)
$$

in which, HEL is the Hugoniot elastic limit and $P_{H E L}$ is the pressure at the $H E L$. The normalized intact and fractured strength are given, respectively, by:

$$
\begin{gathered}
\sigma_{i}^{*}=A\left(P^{*}+T^{*}\right)^{N}\left(1+C \ln \dot{\varepsilon}^{*}\right) \\
\sigma_{f}^{*}=B\left(P^{*}\right)^{M}\left(1+C \ln \dot{\varepsilon}^{*}\right)
\end{gathered}
$$

in which the actual pressure, $P$, and the maximum tensile hydrostatic pressure that the material can withstand, $T$, are normalized respect to $P_{H E L}$. The dimensionless strain rate is $\dot{\varepsilon}^{*}=\dot{\varepsilon} / \dot{\varepsilon}_{0}$, where $\dot{\varepsilon}$ is the actual strain rate and $\dot{\varepsilon}_{0}=1.0 \mathrm{~s}^{-1}$ is the reference strain rate.

The damage variable accumulates with plastic strain according to:

$$
D=\sum\left(\frac{\Delta \varepsilon_{p}}{\Delta \varepsilon_{p}^{f}}\right)
$$

where $\Delta \varepsilon_{p}$ is the plastic strain increment and $\Delta \varepsilon_{p}^{f}$ is the plastic strain at fracture function of the actual pressure,

$$
\varepsilon_{p}^{f}=D_{1}\left(P^{*}+T^{*}\right)^{D_{2}}
$$


Moreover, the hydrostatic behavior is modeled by means of the following equation,

$$
P=K_{1} \mu+K_{2} \mu^{2}+K_{3} \mu^{3}+\Delta P
$$

where, $\mu=\rho / \rho_{0}-1$ is the hydrostatic compression and $\Delta P$ is the bulking pressure of the material that is determined by the amount of the accumulated damage. For the range of pressures investigated in this work, $K_{2}$ and $K_{3}$ can be neglected, while $K_{1}$ is the bulk modulus, $K$, that can be derived by the values of the elastic constants of the material $E$ and $v$.

As a result of the linear EOS, $P_{H E L}$ can be expressed as a function of $H E L$ and two elastic moduli, $K$ and $G$, and then eliminated from the set of unknown parameters, using the following expression:

$$
P_{H E L}=H E L\left(1-\frac{4 G}{3 K+4 G}\right)
$$

Then, in order to completely describe the mechanical behavior of a brittle ceramic with the $\mathrm{JH}-2$ model the following nine parameters have to be determined: $A, B, C$, $M, N, T, H E L, D_{1}, D_{2}$.

\section{Experimental tests}

In order to generate data for the calibration of the $\mathrm{JH}-2$ model parameters, two types of tests were designed and performed:

a) Taylor Cylinder impact test;

b) Drop Weight test.

In the Taylor test, a cylinder, made of the material of interest, is launched, at a given velocity, along its axial axis against a rigid anvil. Input parameters for the test are the impact velocity and the initial geometry of the cylinder. Traditionally, the output of this test is the deformed shape of the impacted cylinder. Deformed profile, diameter of the impact surface and final length are obtained post mortem. In case of breakup, test outputs are the number and the dimensions of fragments. When dealing with brittle materials, such as ceramics, fragments may also be generated from secondary impacts. A clearer insight of the dynamics of fragments formation, as a result of the stress waves travel and interaction, can be better obtained by means of high speed video-recording. To this purpose, it was decided to document each test with the support of high speed camera. This technique allowed the determination of the exact time and location of fragments formation. Taylor tests were performed at the design velocity impact of $\sim 100 \mathrm{~m} / \mathrm{s}$. Since complete rupture of the material occurs at that velocity, slower impact tests were performed in order to determine the threshold impact velocity for which no failure occurs. The nominal diameter of the cylinders was $19 \mathrm{~mm}$ with a length ranging between 90 and $167 \mathrm{~mm}$. A total of 21 tests were performed with impact velocity ranging between 83.0 and $3.0 \mathrm{~m} / \mathrm{s}$ using two different facilities: a gas gun for the higher velocities and a drop weight facility, consisting in a vertical launch pipe with a diameter of $22 \mathrm{~mm}$, for the lower velocities. In Figures 1

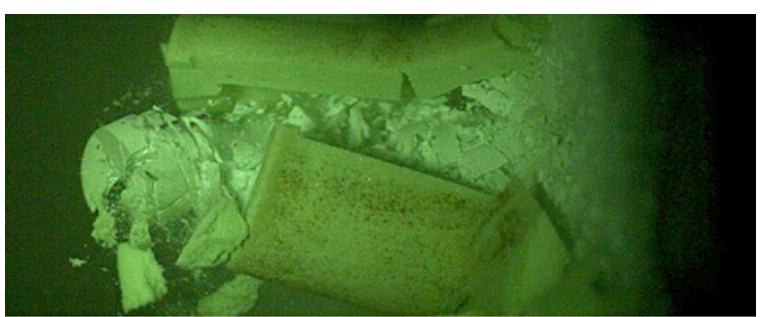

Fig. 1. Fragmentation in Taylor cylinder impacted at high velocity $(\mathrm{v}=83.3 \mathrm{~m} / \mathrm{s})$.

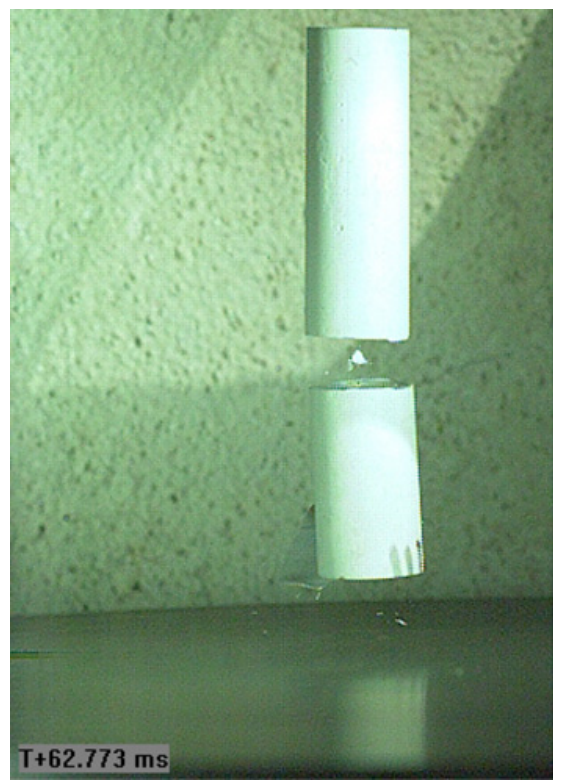

Fig. 2. Spall fracture in Taylor cylinder impacted at low velocity $(\mathrm{v}=4.2 \mathrm{~m} / \mathrm{s})$.

and 2 the high and low impact velocity failure modes are shown.

In the Drop Weight test, a ceramic tile, supported by a rigid plate, is hit by an impactor with hemispherical head of $16.0 \mathrm{~mm}$ diameter. Ceramic tiles were available with different geometries: a rectangular tile with nominal dimensions $L=97.0 \mathrm{~mm}, W=28.0 \mathrm{~mm}, T=4.0 \mathrm{~mm}$, and a square tile with $L=50.0 \mathrm{~mm}$ and $T=8.0 \mathrm{~mm}$. A total of 19 tests were performed with 5 different configurations: rectangular tile at two different velocities, square tile at two different velocities, and another configuration in which two rectangular tiles are stacked. For all tests the Load vs. Displacement curve at the tup of the impactor were recorded providing quantitative data for the $\mathrm{JH}-2$ model parameters calibration. In Figure 3 the response obtained in one test configuration is given showing the two behaviors (high and low peak load) that the material exhibited in all tests.

\subsection{JH-2 model parameters optimization}

All experimental configurations were simulated by means of FEM. Initially, inverse calibration technique was used for the drop weight test configurations. Since for all these type of tests the strain rate is similar, the strain rate 


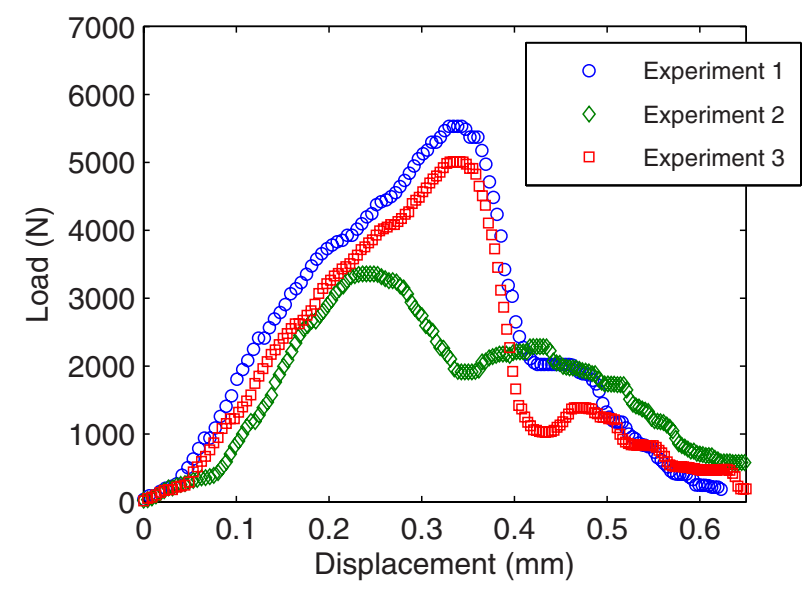

Fig. 3. Load vs. Displacement curve for Drop weight test on rectangular tile at $1.5 \mathrm{~m} / \mathrm{s}$.

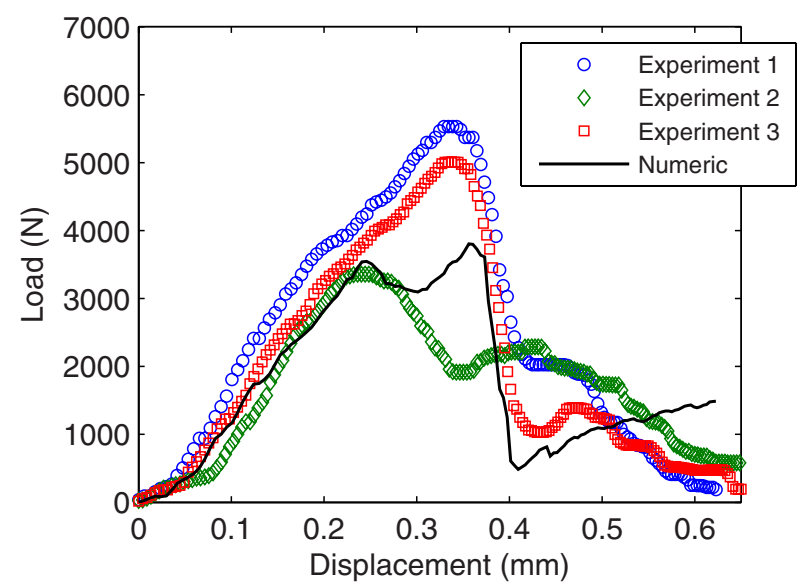

Fig. 4. Comparison between numerical and experimental results for drop weight test on rectangular tile at $1.5 \mathrm{~m} / \mathrm{s}$.

parameter $C$ cannot be determined at this time. All other parameters were optimized minimizing the following objective functions:

obj1 the square root of the summation of the differences between the calculated and the experimental Load vs. Displacement curve (indicated as ErrMin);

obj2 the difference between the numerical and the experimental values of force peak $(F$ delta $)$;

obj3 the difference between the times at which the force peak is reached in the computational and experimental curves ( $\mathrm{T}$ delta);

obj4 the difference between the integrals of the computational and experimental load vs. displacement curve, i.e. the deformation work (IntMin).

The optimization task was carried out in two phases. First, the objectives were minimized separately for each of the 5 configurations using large ranges and large steps for the varying variables; the mean value for each parameter was chosen based on data available for similar material, [2]. A DoE using Sobol algorithm with 38 designs was generated, [3]. The multiobjective genetic algorithm NSGA-II, [4], was used for the optimization with 19 generations for a total of 722 designs for each of the 5 configurations. The 5 data sets found in this way resulted to slightly differ one

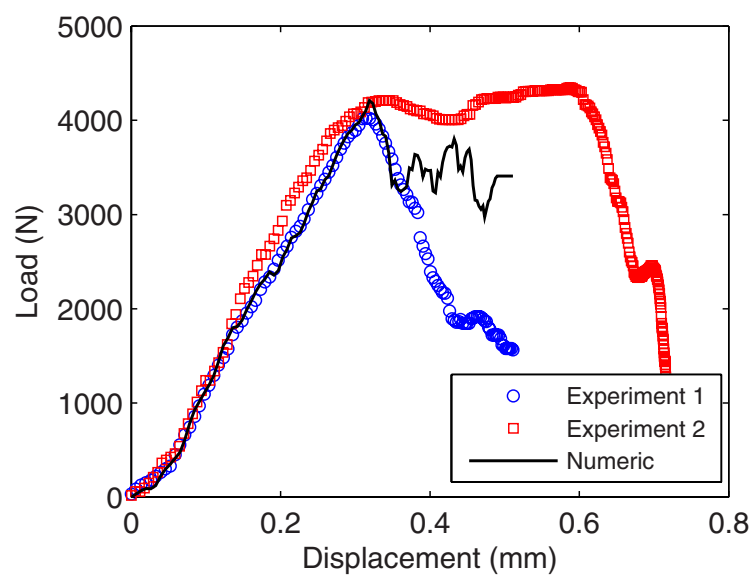

Fig. 5. Comparison between numerical and experimental results for drop weight test on rectangular stacked tiles at $1.1 \mathrm{~m} / \mathrm{s}$.
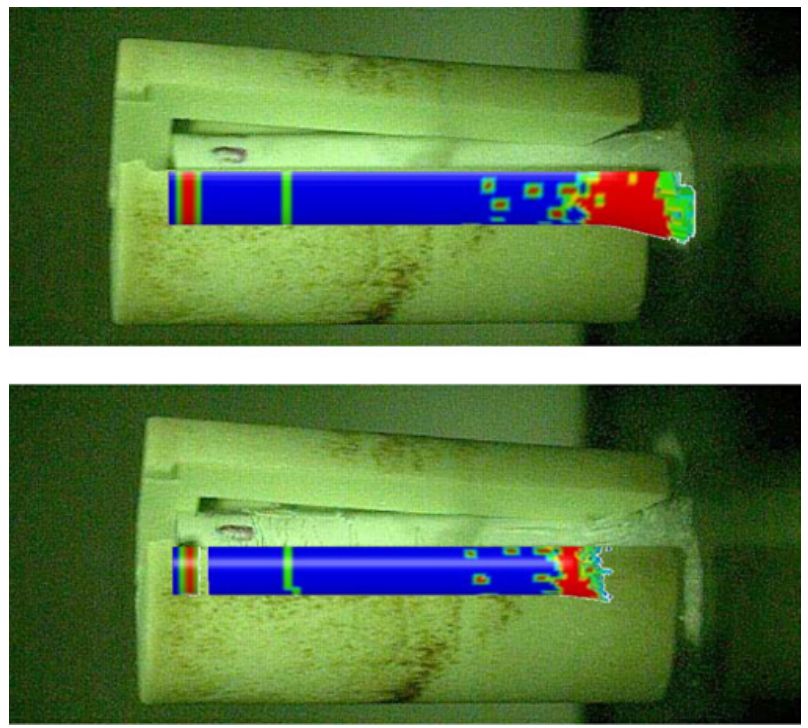

Fig. 6. Qualitative comparison of damage pattern and distribution in Taylor impact at $83.3 \mathrm{~m} / \mathrm{s}$ for two time frames. In the second frame the destroyed material near to impact surface is graphically removed by the erosion algorithm.

from each other. However, this was a necessary step in order to reduce the range and the step of the variables for the second refinement phase of the task.

Successively a new optimization was performed accounting for all configurations at the same time. The analysis (with 20 objective functions) resulted in 1275 finite element simulations, with a total of $1120 \mathrm{~h} \mathrm{CPU} \mathrm{time,}$ that led to the optimized material parameters set whose high-quality performances were demonstrated by the comparison between experimental and numerical results shown in Figures 4 and 5.

Finally, the Taylor impact test configuration was simulated using the set of parameters determined for calibrating the strain rate sensitivity parameter $C$. In this phase the time resolved fragmented length (extracted from the videorecording and shown for comparison in Figure 6) as a function of the impact velocity was used as target function for the determination of $C$. 


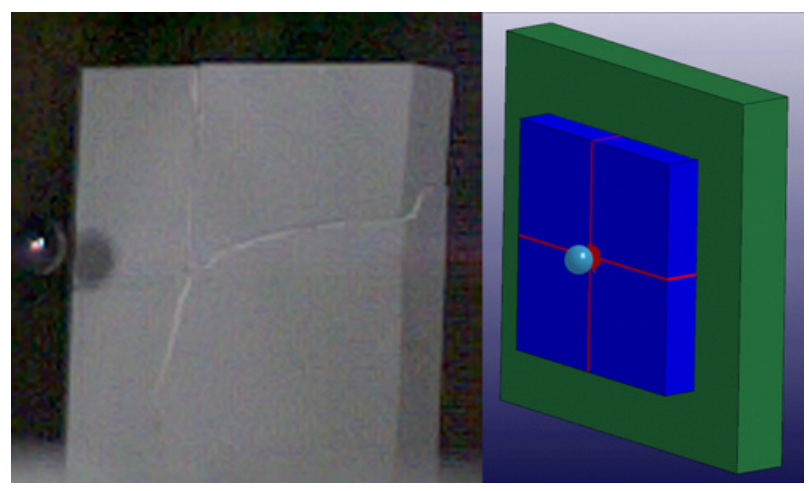

Fig. 7. Qualitative comparison of damage pattern and distribution in the sphere impact test at $30 \mathrm{~m} / \mathrm{s}$.

\subsection{JH-2 model parameters validation}

The determined JH-2 parameters set was verified planning an independent test with a strain rate of $\sim 10^{3} \mathrm{~s}^{-1}$, which is in between that of Drop weight and Taylor test characteristic strain rate values. This test consists in a steel sphere $6.3 \mathrm{~mm}$ in diameter launched against a fused silica tile supported by a rigid plate. Scope of the test is to predict the critical velocity for which failure of the tile occurs and the resulting failure mode.

Running a parametric FEM analysis, it was found that brittle fracture occurs at $30.0 \mathrm{~m} / \mathrm{s}$, while below $20.0 \mathrm{~m} / \mathrm{s}$ no visible damage is expected to occurs. Based on this, two impact tests, respectively at 20.0 and $30.0 \mathrm{~m} / \mathrm{s}$, were planned and performed using a $6.5 \mathrm{~mm}$ gas-gun. Fused silica tile behavior was again monitored using high speed camera. As expected, failure in brittle silica is found to be controlled by the superposition of tensile stress waves, generated by reflection of the compression waves at free surfaces.

As predicted by FEM, high speed video recording confirmed that brittle failure starts to occur approximately
$30.0 \mu \mathrm{s}$ after impact. Fracture occurs as a result of two main cracks running, orthogonally one to each other, from side to side of the tile, Figure 7 . The comparison of numerical and experimental results confirmed the accuracy of the calibrated JH-2 model parameters in predicting failure occurrence under dynamic conditions different than those used in the structural optimization process.

\section{Conclusion}

In this work the determination of the JH-2 mode parameters for fused silica was performed using both quantitative and qualitative data obtained on two different dynamic test configurations. Inverse calibration technique, based on extensive numerical simulation of both type of tests, was implemented. In order to determine the optimum set for the material model parameters multi-objective optimization was used. Finally, an independent validation test was planned and performed. Results seem to indicate that the determined material parameters for the JH-2 model can be confidently used for strain rate ranging from $10^{2}$ to $10^{4} \mathrm{~s}^{-1}$.

\section{References}

1. Johnson G. R. and Holmquist T. J., High-Pressure Science and Technology (American Institute of Physics, 1994)

2. Cronin D. S., Bui K., Kaufmann C., McIntosh G. and Berstad T., Proceedings of the 4th European LS-DYNA Users Conference, (2003) pp. $47-60$

3. Bratley P. and Fox B. L., ACM Transactions on Mathematical Software, 14-1, (1988) 88-100

4. Deb K., Pratap A., Agarwal S. and Meyarivan T., KanGAL Report Number 2000001, (2000) 